Volker Brühl

\title{
Mehr Nachhaltigkeit im deutschen Leitindex DAX
}

\section{Reformvorschläge im Lichte des Wirecard-Skandals}

\author{
Im Rahmen der Aufarbeitung des Wirecard-Skandals wird auch eine Änderung der Kriterien \\ zur Aufnahme in den deutschen Leitindex DAX diskutiert. Die bislang von der Deutschen
} Börse vorgesehenen Maßnahmen gehen in die richtige Richtung, sind aber nicht weitreichend genug. Es bedarf eines deutlichen Zeichens, dass sich künftig nur solche Unternehmen für den DAX qualifizieren können, die ein zumindest befriedigendes Maß an Nachhaltigkeit gemessen durch einen ESG-Risk-Score (Environment, Social, Governance) in ihrer Geschäftstätigkeit erreichen. Eine Simulation verdeutlicht, dass nach ESG-Kriterien seit langem kritisch betrachtete Unternehmen dem DAX nicht mehr angehören würden. Damit könnte mehr Kapital in nachhaltig wirtschaftende Unternehmen und Sektoren fließen.

\begin{abstract}
Der DAX ist das bekannteste deutsche Börsenbarometer und misst die Wertentwicklung der 30 größten und liquidesten Unternehmen des deutschen Aktienmarkts. Er repräsentiert rund $75 \%$ der Marktkapitalisierung und etwa $85 \%$ der Börsenumsätze börsennotierter Gesellschaften in Deutschland (Deutsche Börse, 2018). Der DAX dient als Benchmark für eine Vielzahl von Finanzprodukten, wie ETF (Exchange Traded Funds), die es auch privaten Anleger*innen ermöglichen, etwa im Rahmen der privaten Altersvorsorge in den DAX oder andere Auswahlindizes zu investieren.
\end{abstract}

Darüber hinaus bildet der DAX die Grundlage für zahlreiche derivative Finanzinstrumente, die eine wichtige Rolle bei der Risikoabsicherung von institutionellen Anleger*innen und Unternehmen spielen. Neben dem DAX umfasst die DAX-Indexfamilie weitere Indizes wie den MDAX, der die 60 größten und börsenumsatzstärksten Unternehmen unterhalb des DAX abbildet, den TecDAX, dem Technologieunternehmen angehören, und den SDAX, dem die 70 größten und meistgehandelten Unternehmen unterhalb des MDAX angehören (Deutsche Börse, 2019). Der deut-

(C) Der/die Autor(en) 2020. Open Access: Dieser Artikel wird unter der Creative Commons Namensnennung 4.0 International Lizenz (https:// creativecommons.org/licenses/by/4.0/deed.de) veröffentlicht.

Open Access wird durch die ZBW - Leibniz-Informationszentrum Wirtschaft gefördert.

Prof. Dr. Volker Brühl ist Geschäftsführer am Center for Financial Studies an der Goethe-Universität in Frankfurt am Main. sche Leitindex DAX wurde am 1. Juli 1988 von der Deutsche Börse $A G$ eingeführt mit einem Referenzwert von 1.000 Punkten (Deutsche Börse, 2019).

In den vergangenen Monaten hat der Aufstieg und Fall des Zahlungsdienstleisters Wirecard AG mit Sitz in Aschheim vielfache Diskussionen ausgelöst über mögliche Unzulänglichkeiten bei der Bundesanstalt für Finanzdienstleistungsaufsicht (BaFin), die Verantwortung der zuständigen Wirtschaftsprüfer*innen und schließlich auch über die Kriterien für eine Aufnahme in den deutschen Leitindex DAX. Wirecard wurde lange Zeit als ein Vorzeigeunternehmen gefeiert, schien Wirecard doch endlich ein deutsches Finanztechnologieunternehmen von internationalem Rang zu sein. Schließlich verdrängte Wirecard die Commerzbank am 24. September 2018 aus dem DAX. Mit einem Börsenwert von bis zu 25 Mrd. Euro war Wirecard zeitweise höher bewertet als die Deutsche Bank.

Anhaltende Gerüchte über gefälschte Zahlen, Scheingeschäfte und nicht existierende Bankguthaben führten schließlich zu einer Sonderprüfung der Bilanzen von Wirecard und zur Verweigerung des uneingeschränkten Bestätigungsvermerks durch die zuständige Wirtschaftsprüfungsgesellschaft. Letztlich kam es zum Zusammenbruch des einstigen Börsen-Stars, der am 25. Juni 2020 einen Insolvenzantrag stellen musste. Trotzdem dauerte es noch bis zum 21. August 2020, bis Wirecard offiziell aus dem DAX ausscheiden konnte, da die Index-Regularien der Deutschen Börse ein unmittelbares Ausscheiden bei einem Insolvenzantrag zu diesem Zeitpunkt nicht vorsahen.

Die Insolvenz eines DAX-Unternehmens ist ein schwerer Schlag für das Vertrauen vor allem von Privatanleger*innen 
in die Integrität der Kapitalmärkte. Zum einen wird der DAX als eine Gruppe von sogenannten Blue-Chip-Unternehmen positioniert, die besonders hohe Qualitätsstandards erfüllen. Daher fühlen sich auch Privatanleger*innen mit sonst geringer Affinität zu Aktien bei solchen Aktien gut aufgehoben, wo man zwar mit Kursgewinnen und -verlusten rechnen muss, jedoch kaum von einem Totalverlust durch eine Insolvenz ausgeht. Zum anderen gibt es zahlreiche Aktienfonds, die den DAX als Index Tracker nachbilden und dadurch auch Privatanleger*innen die Möglichkeit geben, in ein diversifiziertes und damit risikoärmeres Aktienportfolio zu investieren. Der verzögerte Ausschluss von Wirecard aus dem DAX nach der Insolvenz hatte zur Folge, dass vor allem den DAX nachbildende ETFs diese Aktie im Portfolio halten mussten, obwohl sie sich sonst vermutlich zur Verlustbegrenzung sofort von diesem Wert getrennt hätten.

Ein weiterer Anlass für eine kritische Betrachtung der derzeitigen Konstruktion des DAX war die Nominierung des Unternehmens Delivery Hero SE als Nachfolger von Wirecard im DAX ab dem 24.8.2020. Denn das Unternehmen hat in den vergangenen Jahren weder einen operativen Gewinn noch ein positives Jahresergebnis erzielt. Gerade dieser Umstand wirft die Frage auf, ob das Geschäftsmodell dieses Unternehmens über die erforderliche Qualität und Nachhaltigkeit verfügt, die man von einem DAX-Unternehmen erwarten kann. Außerdem hat das Unternehmen seit dem Verkauf des Deutschlandgeschäfts an den niederländischen Konkurrenten Just Eat Takeaway (Lieferando) keinerlei Geschäftsaktivitäten mehr in Deutschland. Dies gab es in der Geschichte des DAX auch noch nie.

Es stellt sich die Frage, ob die derzeitigen Kriterien für eine Zugehörigkeit zum DAX, die ausschließlich auf dem Börsenwert der nicht im Festbesitz befindlichen Aktien (Free Float) und den Handelsumsätzen basieren, noch zeitgemäß sind oder ob diese nicht modifiziert werden müssten, um dem Anspruch eines Blue-Chip-Index auch künftig genügen zu können. Die Deutsche Börse hat kürzlich eine Marktkonsultation durchgeführt, die mit einem ausgewählten Fragenkatalog die Meinung von Marktteilnehmer*innen eingeholt hat. Es werden sowohl diese Vorschläge diskutiert als auch die immer wichtiger werdenden Nachhaltigkeitsaspekte, die bei einer Reformierung des DAX Berücksichtigung finden sollten. Wie ESG-Aspekte in die Neugestaltung des DAX einfließen können und welche Auswirkungen dies auf die gegenwärtige Zusammensetzung des DAX haben könnte, wird ebenfalls erörtert.

\section{Konstruktion des DAX}

Der DAX bildet die Wertentwicklung der 30 gemessen an der Marktkapitalisierung größten und gemessen am
Börsenhandelsumsatz liquidesten Aktien in Deutschland ab. Alle Auswahlindizes der DAX-Index-Familie werden als Kurs-Indizes (bilden die reine Kursentwicklung ab), als Performance-Indizes (berücksichtigt die vollständige Reinvestition der Dividenden) und als Net-Return-Indizes (berücksichtigt die steuerbereinigte Reinvestition der Dividenden) berechnet. Der DAX wird üblicherweise in der Variante des Performance-Index betrachtet.

Absolute Höhe und Veränderungen des DAX werden vor allem in der Öffentlichkeit als Indikator für den Zustand und die Aussichten der deutschen Wirtschaft verstanden. Diese Interpretation ist aus verschiedenen Gründen zu weitgehend. Denn aufgrund des gerade in Deutschland relativ geringen Anteils börsennotierter Unternehmen an der Gesamtzahl der deutschen Unternehmen bildet der DAX die stark mittelständisch geprägte industrielle Landschaft nicht repräsentativ ab. Außerdem werden die Kursentwicklungen von Unternehmen nicht nur durch die jeweilige Geschäftssituation und die Erwartung des Marktes über die künftige Geschäftsentwicklung getrieben, sondern auch von einer Vielzahl psychologischer Faktoren, die das Anlegerverhalten beeinflussen. Jedoch repräsentiert der DAX durchaus das Sentiment auf den deutschen Märkten, da er ca. $75 \%$ der Börsenwerte sämtlicher deutscher börsennotierter Gesellschaften abbildet. Die Zusammensetzung des DAX wird wie die der übrigen Aktienindizes der Deutsche Börse Gruppe quartalsweise überprüft. Der Auswahlprozess ist vollständig regelbasiert und soll so für Vertrauen, Transparenz und Nachbildbarkeit bei den Anleger ${ }^{*} i n n e n$ sorgen. Dies unterscheidet den DAX z. B. vom Dow Jones Industrial Average-Index (DJIA), bei dem die Auswahl der Index-Konstituenten nicht regelbasiert, sondern von dem zuständigen Index-Committee nach qualitativen, nicht immer transparent nachvollziehbaren Kriterien erfolgt (S\&P Dow Jones Indices, 2019).

Für eine Aufnahme in den DAX qualifizieren sich Unternehmen nach folgenden Kriterien:

- Orderbuchumsatz in Xetra und am Parkett Frankfurt (in den vorangegangenen zwölf Monaten)

- Free-Float-Marktkapitalisierung zu einem bestimmten Stichtag (letzter Handelstag im Monat). Diese wird aus dem durchschnittlichen volumengewichteten Durchschnittspreis (VWAP) der vergangenen 20 Handelstage ermittelt.

- Erfüllung der Transparent-Anforderungen im Prime Standard

- Streubesitzanteil von mindestens $10 \%$

- Juristischer Sitz oder operatives Hauptquartier in Deutschland

- Fortlaufender Handel auf Xetra

- Mindestens 30 Handelstage seit Erstnotiz 
Das Gewicht eines Mitglieds im DAX ist auf höchstens $10 \%$ beschränkt, um zu verhindern, dass einzelne Unternehmen den Index dominieren.

Einmal jährlich im September findet eine ordentliche Anpassung des DAX statt. Zusätzlich findet jeweils zum Ende der übrigen Quartale eine Überprüfung der Indexzusammensetzung auf Basis der sogenannten Fast-Entryund Fast-Exit-Regeln statt. Eine solche Indexanpassung dient dazu, signifikanten Verschiebungen auf der Rangliste der größten börsennotierten Gesellschaften Rechnung zu tragen, die beispielsweise durch Börsengänge, Fusionen und Übernahmen oder große Kapitalmaßnahmen entstehen können. Umgekehrt können starke Kursrückgänge durch Geschäftseinbrüche oder andere Faktoren einen zeitnahen Ausschluss aus dem DAX nahelegen.

Eine regelbasierte Vorgehensweise bei der Indexzusammensetzung erfolgt ebenfalls bei den sogenannten StoxxIndizes, von denen der Euro Stoxx 50 oder der Stoxx 600 Europe beispielhaft erwähnt seien. Die Index-Familie Stoxx gehört ebenfalls zur Deutsche Börse Gruppe und stellt eine Vielzahl von nach geografischen, sektoralen oder sonstigen Kriterien erstellten Aktienindizes bereit (Qontigo, 2020a; Qontigo, 2020b).

\section{Marktkonsultation der Deutschen Börse}

Die Deutsche Börse Gruppe hat eine Marktkonsultation zu den Auswahlindizes DAX, MDAX, SDAX und TecDAX mit dem Ziel initiiert, striktere Kriterien bei der Aufnahme in die betreffenden Indizes einzuführen. Dadurch soll eine Angleichung an internationale Index-Standards unter Beibehaltung der Transparenz, Berechenbarkeit und Objektivität eines regelbasierten Rahmens erreicht werden (Deutsche Börse, 2020). Die Konsultation stand allen Marktteilnehmer*innen vom 5. Oktober 2020 bis zum 4. November 2020 offen. Die Ergebnisse der Marktkonsultation wurden am 24. November 2020 veröffentlicht. Die beschlossenen Veränderungen sollen sukzessive umgesetzt werden. Nachfolgend werden die wesentlichen Maßnahmen der Deutschen Börse mit Blick auf den DAX aufgeführt und im Lichte internationaler Standards diskutiert.

- Erweiterung des DAX auf insgesamt 40 Werte. Die VergröBerung des DAX ist eher kritisch zu betrachten, da durch die weitere Steigerung der ohnehin schon hohen Marktabdeckung die kleineren Indizes ausgedünnt werden.

- Abschaffung der Voraussetzung Prime Standard für die Aufnahme in den DAX. Der Prime Standard stellt ein Marktsegment der Deutschen Börse dar, das besonders hohe Transparenzanforderungen an die dort notierten Unternehmen stellt, die deutlich über den so- genannten General Standard hinausgehen, in dem die gesetzlichen Anforderungen für den regulierten Markt gelten. Bislang können nur solche Unternehmen in einen Index aufgenommen werden, die am regulierten Markt notieren und zusätzlich die erhöhten Transparenzkriterien des Prime Standard erfüllen. Diese Voraussetzung wird abgeschafft, sodass künftig auch Unternehmen des General Standard in einen Index aufgenommen werden können. Begründet wird dies mit einer höheren Flexibilität bei der Festlegung der IndexRegelung, wenn man eine Index-Zugehörigkeit von den Listing-Segmenten entkoppelt. Dagegen spricht, dass gerade Index-Unternehmen einem besonders hohen Qualitätsstandard genügen müssen und dazu auch besondere Transparenz- und Berichtspflichten gehören. Ähnlich hohe Anforderungen sind z. B. bei britischen Indizes wie dem FTSE 100 zu erfüllen, in den nur sogenannte Premium Listed Equity Shares aufgenommen werden (FTSE Russel, 2020). Der Verzicht auf die besonderen Anforderungen des Prime Standard ist daher ein falsches Signal.

- Ersatz des Selektionskriteriums Börsenumsatz durch Mindestliquidität. Bislang wird neben der Marktkapitalisierung des Free Float auch die Rangfolge des Unternehmens auf der Grundlage der 12-Monats-Börsenumsätze berücksichtigt. Künftig soll stattdessen eine Mindestliquidität während des vierteljährlichen Index Review ausgewertet werden. Zur Bewertung wird die Umschlagshäufigkeit als Quotient aus dem 12-Monatsumsatz und der (Free Float-) Marktkapitalisierung berechnet. Für die Aufnahme in einen Index ist eine Umschlagshäufigkeit von 0,2 oder ein absoluter 12-Monatsumsatz von 1 Mrd. Euro erforderlich. Zum Verbleib in einem Index ist eine Umschlagshäufigkeit von 0,1 oder 0,8 Mrd. Euro erforderlich (Deutsche Börse, 2020). Diese Veränderung der Selektionsmethodik ist nachvollziehbar, da es international üblich ist, als Selektionskriterien die Marktkapitalisierung (insgesamt oder des Free Float) heranzuziehen. Die Sicherung einer ausreichenden Handelbarkeit der Index-Unternehmen kann durch eine Mindestliquidität gewährleistet werden. Die Vergleichbarkeit des DAX mit anderen internationalen Benchmarks wie z. B. dem Dow Jones Industrial Average, dem S\&P 500 oder dem FTSE 100 wird auf diese Weise erleichtert.

- Fast Exit bei nicht fristgerechter Vorlage eines testierten Jahresberichts. Die Empfehlung F.2 des Deutschen Corporate Governance Kodex sieht vor, dass der Konzernabschluss und der -lagebericht binnen 90 Tagen nach Geschäftsjahresende sowie die verpflichtenden unterjährigen Finanzinformationen binnen 45 Tagen nach Ende des Berichtszeitraums öffentlich zugänglich 
sein müssen (Regierungskommission, 2020). Künftig sollen Unternehmen, die gegen diese Bestimmungen verstoßen, innerhalb von zwei Werktagen aus dem Index gestrichen werden, wenn der entsprechende Bericht nicht innerhalb der nächsten 30 Tage veröffentlicht wird. Ein Unternehmen wird auch in einem Auswahlindex ersetzt, wenn es selbst oder seine Wirtschaftsprüfung die jeweiligen testierten Jahresabschlüsse nach der Veröffentlichung zurückzieht, sobald die Frist überschritten wurde. Dieser Vorschlag ist eine direkte Konsequenz aus dem Wirecard-Skandal und ermöglicht künftig einen Ausschluss aus dem jeweiligen Index, sofern die entsprechenden Pflichten verletzt werden. Dies ist eindeutig zu begrüßen.

- Verpflichtende Einsetzung eines Prüfungsausschusses im Aufsichtsrat. Künftig sollen Unternehmen von Auswahlindizes die Empfehlungen des Deutschen Corporate Governance Kodex in Bezug auf den Prüfungsausschuss zwingend erfüllen, um eine besonders hohe Qualität im Bereich Corporate Governance zu gewährleisten. Auch dieser Vorschlag ist eine Folge der Unzulänglichkeiten von Wirecard. Denn Wirecard hat erst 2019 einen Prüfungsausschuss eingerichtet, sodass zumindest die Möglichkeiten zur Kontrolle des Jahresabschlusses bis zu diesem Zeitpunkt unzureichend waren. Auch wenn dies grundsätzlich nachvollziehbar ist, greift diese Maßnahme zu kurz. Man sollte künftig zumindest bei DAXUnternehmen als Voraussetzung einführen, dass der Deutsche Corporate Governance Kodex vollumfänglich zu erfüllen ist. Sonst sollte keine Aufnahme in den DAX bzw. bei Verstößen ein Fast Exit aus dem DAX erfolgen.

- Profitabilität des Unternehmens als Aufnahmekriterium. Für die erstmalige Aufnahme in den DAX müssen die Unternehmen in beiden zuletzt veröffentlichten Geschäftsberichten zum Zeitpunkt der Index-Aufnahme ein positives EBITDA (Earnings Before Interest, Taxes, Depreciation and Amortization) aufweisen. Das EBITDA ist eine international übliche Kennzahl für die Messung der Profitabilität des operativen Geschäfts, die vor Zinsergebnis, Ertragsteuern, Abschreibungen und Amortisationen berechnet wird, um eine bessere internationale Vergleichbarkeit zu erreichen. Diese Anforderung betrifft solche Unternehmen nicht, die zum Zeitpunkt der Einführung der Regel bereits Bestandteil des Index sind. Diese Veränderung geht nicht weit genug und ist zu unpräzise formuliert. Andere internationale Indizes definieren die Qualität eines Unternehmens unter anderem auch über seine Fähigkeit, nachhaltig profitabel zu arbeiten - und dies nicht nur zum Zeitpunkt der Aufnahme in den DAX. Daher sollte zum einen präzisiert werden, wie genau das relevante EBITDA zu ermitteln ist. Insbesondere sollte es sich um ein von außeror- dentlichen und periodenfremden Effekten bereinigtes EBITDA handeln und von der Wirtschaftsprüfung des Unternehmens bestätigt werden. Zum anderen sollte es zu einem Ausschluss von Unternehmen aus dem DAX kommen, falls das Unternehmen über einen längeren Zeitraum (z.B. acht Quartale) hintereinander kein positives operatives Ergebnis erwirtschaftet. Ausnahmen von dieser Regel könnten von der Deutschen Börse dann zugelassen werden, wenn die betreffenden Unternehmen durch eine länger anhaltende tiefe Rezession der Gesamtwirtschaft nicht profitabel sein können und dies demzufolge kein unternehmensspezifisches Problem widerspiegelt.

- Einführung eines Ausschlusskriteriums für Unternehmen, die sich an bestimmten Waffengeschäften beteiligen (Controversial Weapons). DAX-Unternehmen sollten künftig nicht an Produktion oder Vertrieb von „umstrittenen Waffen“ mitwirken dürfen. Die folgenden Waffenkategorien sollten diesem Kriterium unterliegen: Antipersonenminen, biologische und chemische Waffen, Streuwaffen, angereichertes Uran, Atomwaffen und Waffen mit weißem Phosphor. Dieser Vorschlag wird aufgrund des heterogenen Feedbacks der Marktteilnehmenden nicht umgesetzt. Allerdings greift diese Überlegung ohnehin zu kurz.

Von einem DAX-Unternehmen kann man erwarten, dass es höchste Standards bei allen wesentlichen Nachhaltigkeitskriterien erfüllt. In der Finanzindustrie wird Nachhaltigkeit anhand von drei Dimensionen definiert und gemessen, die man unter ESG zusammenfasst (MSCI, 2020). Daher wäre es sinnvoll, wenn künftig jedes DAX-Unternehmen ein bestimmtes ESG-Risikopotenzial - gemessen durch einen ESG Risk Score - nicht überschreiten darf und bei Nichterfüllung über einen gewissen Zeitraum ein Ausschluss erfolgen kann. Inzwischen gibt es mehrere etablierte Ratingagenturen, die sich auf die Messung von Nachhaltigkeitsrisiken spezialisiert haben. Dazu gehören z. B. Sustainalytics, MSCI und ISS-Oekom. Die Deutsche Börse selbst hat im März dieses Jahres mit dem DAX 50 ESG einen neuen Index eingeführt, bei dem in die Selektion der Index-Unternehmen neben den beiden beim klassischen DAX angewendeten Kriterien Börsenwert und Börsenumsatz auch ESG-Kriterien einfließen. Auch wenn die Einführung neuer Indizes unter stärkerer Berücksichtigung von Nachhaltigkeitskriterien wünschenswert ist, so ersetzt dies nicht eine entsprechende Reform der jeweiligen Leitindizes wie hierzulande des DAX.

\section{Vorschlag für einen nachhaltigen DAX}

Um Nachhaltigkeitsaspekte auch beim DAX umfassend zu berücksichtigen, bietet es sich an, eine Aufnahme 
Tabelle 1

Mögliche Veränderungen im DAX durch Einführung von ESG-Risk-Score, Mindestliquidität und Profitabilität

\begin{tabular}{|c|c|c|c|c|c|c|c|c|c|c|}
\hline ISIN & Unternehmen & $\begin{array}{l}\text { Derzeiti- } \\
\text { ger Index }\end{array}$ & $\begin{array}{c}\text { Rang } \\
\text { Market Cap }\end{array}$ & $\begin{array}{l}\text { Rang Bör- } \\
\text { senumsatz }\end{array}$ & $\begin{array}{l}\text { ESG Risk } \\
\text { Score }\end{array}$ & $\begin{array}{c}\text { ESG- } \\
\text { Risikoklasse }\end{array}$ & $\begin{array}{l}\text { Min. ESG } \\
\text { Score }\end{array}$ & $\begin{array}{l}\text { Profita- } \\
\text { bilität }\end{array}$ & $\begin{array}{l}\text { Neuer } \\
\text { Index }\end{array}$ & $\begin{array}{l}\text { Verände- } \\
\text { rung Index }\end{array}$ \\
\hline DE0007164600 & SAP & DAX & 1 & 1 & 12,9 & low & $\sqrt{ }$ & $\sqrt{ }$ & DAX & $\rightarrow$ \\
\hline IE00BZ12WP82 & LINDE & DAX & 2 & 7 & 11,9 & low & $\sqrt{ }$ & $\sqrt{ }$ & DAX & $\rightarrow$ \\
\hline DE0007236101 & SIEMENS & DAX & 3 & 3 & 29,1 & medium & $\sqrt{ }$ & $\sqrt{ }$ & DAX & $\rightarrow$ \\
\hline DE0008404005 & ALLIANZ & DAX & 4 & 2 & 16,2 & low & $\sqrt{ }$ & $\sqrt{ }$ & DAX & $\rightarrow$ \\
\hline DE000BAY0017 & BAYER & DAX & 5 & 4 & 34,4 & high & - & $\sqrt{ }$ & MDAX & $\downarrow$ \\
\hline DE000A1EWWWO & ADIDAS & DAX & 6 & 8 & 13,9 & low & $\sqrt{ }$ & $\sqrt{ }$ & DAX & $\rightarrow$ \\
\hline DE000BASF111 & BASF & DAX & 7 & 5 & 28,5 & medium & $\sqrt{ }$ & $\sqrt{ }$ & DAX & $\rightarrow$ \\
\hline DE0005557508 & DEUTSCHE TELEKOM & DAX & 8 & 10 & 16,7 & low & $\sqrt{ }$ & $\sqrt{ }$ & DAX & $\rightarrow$ \\
\hline NL0000235190 & AIRBUS & MDAX & 9 & 47 & 30,9 & high & - & $\sqrt{ }$ & MDAX & $\rightarrow$ \\
\hline DE0005552004 & DEUTSCHE POST & DAX & 10 & 14 & 16,0 & low & $\sqrt{ }$ & $\sqrt{ }$ & DAX & $\rightarrow$ \\
\hline DE0007100000 & DAIMLER & DAX & 11 & 6 & 25,6 & medium & $\sqrt{ }$ & $\sqrt{ }$ & DAX & $\rightarrow$ \\
\hline DE0008430026 & MUENCH. RUECKVERS. & DAX & 12 & 12 & 18,1 & low & $\sqrt{ }$ & $\sqrt{ }$ & DAX & $\rightarrow$ \\
\hline DE000A1ML7J1 & VONOVIA & DAX & 13 & 21 & 7,7 & negl. & $\sqrt{ }$ & $\sqrt{ }$ & DAX & $\rightarrow$ \\
\hline DE0006231004 & INFINEON TECH & DAX & 14 & 11 & 17,8 & low & $\sqrt{ }$ & $\sqrt{ }$ & DAX & $\rightarrow$ \\
\hline DE0005810055 & DEUTSCHE BOERSE & DAX & 15 & 18 & 13,8 & low & $\sqrt{ }$ & $\sqrt{ }$ & DAX & $\rightarrow$ \\
\hline DE0007664039 & VOLKSWAGEN & DAX & 16 & 9 & 41,1 & severe & - & $\sqrt{ }$ & MDAX & $\downarrow$ \\
\hline DE000ENAG999 & E.ON & DAX & 17 & 17 & 23,7 & medium & $\sqrt{ }$ & $\sqrt{ }$ & DAX & $\rightarrow$ \\
\hline DE0007037129 & RWE & DAX & 18 & 16 & 36,2 & high & - & $\sqrt{ }$ & MDAX & $\downarrow$ \\
\hline DE0005190003 & BMW & DAX & 19 & 15 & 27,1 & medium & $\sqrt{ }$ & $\sqrt{ }$ & DAX & $\rightarrow$ \\
\hline DE0005785604 & FRESENIUS & DAX & 20 & 20 & 19,6 & low & $\sqrt{ }$ & $\sqrt{ }$ & DAX & $\rightarrow$ \\
\hline DE0006599905 & MERCK & DAX & 21 & 25 & 19,4 & low & $\sqrt{ }$ & $\sqrt{ }$ & DAX & $\rightarrow$ \\
\hline DE0005140008 & DEUTSCHE BANK & DAX & 22 & 13 & 29,9 & medium & $\sqrt{ }$ & $\sqrt{ }$ & DAX & $\rightarrow$ \\
\hline DE0006048432 & HENKEL & DAX & 23 & 28 & 20,5 & medium & $\sqrt{ }$ & $\sqrt{ }$ & DAX & $\rightarrow$ \\
\hline DE000SYM9999 & SYMRISE & MDAX & 24 & 38 & 24,6 & medium & $\sqrt{ }$ & $\sqrt{ }$ & DAX & $\uparrow$ \\
\hline DE0005785802 & FRESEN. MED. CARE & DAX & 25 & 23 & 18,8 & low & $\sqrt{ }$ & $\sqrt{ }$ & DAX & $\rightarrow$ \\
\hline DE000A0HN5C6 & DEUTSCHE WOHNEN & DAX & 26 & 31 & 18,0 & low & $\sqrt{ }$ & $\sqrt{ }$ & DAX & $\rightarrow$ \\
\hline DE000ZAL1111 & ZALANDO & MDAX & 27 & 40 & 11,7 & low & $\sqrt{ }$ & $\sqrt{ }$ & DAX & $\uparrow$ \\
\hline DE000A2E4K43 & DELIVERY HERO & DAX & 28 & 32 & 25,3 & medium & $\sqrt{ }$ & - & MDAX & $\downarrow$ \\
\hline DE0007165631 & SARTORIUS & MDAX & 29 & 50 & 33,1 & high & - & $\sqrt{ }$ & MDAX & $\rightarrow$ \\
\hline DE0005439004 & CONTINENTAL & $\mathrm{DAX}$ & 30 & 22 & 13,1 & low & $\sqrt{ }$ & $\sqrt{ }$ & DAX & $\rightarrow$ \\
\hline NL0012169213 & QIAGEN & MDAX & 31 & 33 & 17,5 & low & $\sqrt{ }$ & $\sqrt{ }$ & DAX & $\uparrow$ \\
\hline DE0005200000 & BEIERSDORF & DAX & 32 & 30 & 23,4 & medium & $\sqrt{ }$ & $\sqrt{ }$ & DAX & $\rightarrow$ \\
\hline DE000LEG1110 & LEG IMMOBILIEN & MDAX & 33 & 45 & 20,1 & medium & $\sqrt{ }$ & $\sqrt{ }$ & DAX & $\uparrow$ \\
\hline DE000A1DAHHO & BRENNTAG AG & MDAX & 34 & 52 & 20,5 & medium & $\sqrt{ }$ & $\sqrt{ }$ & DAX & $\uparrow$ \\
\hline DE000SHL1006 & SIEMENS HEALTH & MDAX & 35 & 35 & 37,5 & high & - & $\sqrt{ }$ & MDAX & $\rightarrow$ \\
\hline DE0008402215 & HANNOVER RUECK & MDAX & 36 & 43 & 18,5 & low & $\sqrt{ }$ & $\sqrt{ }$ & DAX & $\uparrow$ \\
\hline DE000A0D9PT0 & MTU AERO ENGINES & DAX & 37 & 24 & 23,8 & medium & $\sqrt{ }$ & $\sqrt{ }$ & DAX & $\rightarrow$ \\
\hline DE0006047004 & HEIDELBERGCEMENT & DAX & 38 & 29 & 29,5 & medium & $\sqrt{ }$ & $\sqrt{ }$ & MDAX & $\downarrow$ \\
\hline DE000A12DM80 & SCOUT24 & MDAX & 39 & 49 & 20,0 & medium & $\sqrt{ }$ & $\sqrt{ }$ & MDAX & $\rightarrow$ \\
\hline DE0006062144 & COVESTRO & DAX & 40 & 26 & 20,0 & medium & $\sqrt{ }$ & $\sqrt{ }$ & MDAX & $\downarrow$ \\
\hline
\end{tabular}

Quelle: eigene Darstellung, Deutsche Börse, Sustainalytics.

in den DAX davon abhängig zu machen, dass der in einem ESG-Rating erzielte Punktwert ein höchstens mittleres ESG-Risiko reflektiert. Nachfolgend nutzen wir die Rating-Methodik von Sustainalytics, die mit Hilfe eines Scoring-Modells das Risiko eines Unternehmens durch ESG-Faktoren einschätzt. Dabei wird jedes Unternehmen je nach Sektor anhand einer Vielzahl von Kriterien absolut und in Relation zu einer Vergleichsgruppe von Unternehmen aus derselben Industrie bewertet. Aus der gewich- teten Summe der einzelnen Risikoparameter wird ein aggregierter ESG Risk Score ermittelt, der dann zu einer Einordnung der Unternehmen in eine von insgesamt fünf ESG-Risikoklassen führt. Dabei wird sowohl die Höhe als auch die Beeinflussbarkeit der ESG-Risiken berücksichtigt, denen das Unternehmen ausgesetzt ist, und wie gut der beeinflussbare Teil des Risikos durch das Unternehmen mitigiert wird. Eine Beziehung zwischen den jeweiligen Scores und der Höhe eines damit verbundenen fi- 
nanziellen Verlustrisikos besteht nicht. Es handelt sich um eine Ordinalskala, die ähnlich wie bei den von den großen Kredit-Ratingagenturen (S\&P, Moody's, Fitch) verwendeten Bonitätsnoten, nur eine Rangfolge darstellt.

Folgende ESG-Risikoklassen unterscheidet die RatingMethodik von Sustainalytics (2020): Negligible Risk: overall score of 0-9.99 points, Low Risk: 10-19.99 points, Medium Risk: 20-29.99 points, High Risk: 30-39.99 points, Severe Risk: 40 and higher points. Auf der Basis der hier exemplarisch verwendeten Rating-Methodik von Sustainalytics wird vorgeschlagen, künftig nur noch solche Unternehmen in den DAX aufzunehmen bzw. dort zu belassen, die höchstens einen mittleren ESG Risk Score aufweisen. Dies würde bedeuten, dass alle Unternehmen mit einem ESG Risk Score von High Risk oder Severe Risk dem DAX nicht mehr angehören würden.

Tabelle 1 verdeutlicht, dass eine solche stärker auf Nachhaltigkeit ausgelegte Reformierung des DAX in Verbindung mit der Bedingung, dass Unternehmen im DAX profitabel sein müssen, zu erheblichen Verschiebungen im DAX führen würde. Es sei an dieser Stelle betont, dass die in Tabelle 1 abgebildeten Veränderungen im DAX nur eine aus Sicht des Autors plausible Szenario-Rechnung darstellen. Dennoch zeigt sie interessante Tendenzen auf. Basierend auf den Daten zu Börsenwerten und -umsätzen, die den von der Deutschen Börse veröffentlichten Ranglisten zum 30.9.2020 zugrunde liegen, zeigt Tabelle 1 die gegenwärtige Zusammensetzung des DAX. Legt man das hier vorgeschlagene Mindestniveau an Nachhaltigkeit als weiteres Kriterium zugrunde, würden prominente Namen wie BAYER, Volkswagen und RWE nicht mehr dem DAX angehören. Statt derer würden die Unternehmen Symrise, Zalando und Qiagen in den DAX aufrücken. Delivery Hero - gerade in den DAX eingezogen - würde ebenfalls wegen fehlender Profitabilität aus dem DAX ausscheiden. Außerdem würden sich die Unternehmen HeidelbergCement und Covestro nicht mehr für den DAX qualifizieren, wenn der Börsenwert des Free Float das wichtigste Selektionskriterium in Verbindung mit der
Erfüllung einer Mindestliquidität wäre, obwohl sie sowohl die Kriterien Nachhaltigkeit und Profitabilität erfüllen würden. Dafür würden die Unternehmen LEG Immobilien, Brenntag und Hannover Rück dem DAX angehören.

\section{Fazit}

Die bislang von der Deutschen Börse vorgelegten Maßnahmen zur Reformierung des DAX (und anderer Indizes) gehen nicht weit genug. Es bedarf eines deutlichen Zeichens, dass sich nur solche Unternehmen für den DAX qualifizieren dürfen, die ein zumindest befriedigendes $\mathrm{Maß}$ an Nachhaltigkeit erreichen. Dies könnte man über einen ESG Risk Score erfassen. Die Daten werden von qualifizierten, auf Nachhaltigkeit spezialisierten Ratingagenturen bereits heute detailliert erfasst und sind daher sofort verfügbar. Die vorgestellte Simulation verdeutlicht, dass unter ESG-Kriterien seit langem kritisch betrachtete Unternehmen sich nicht mehr für den DAX qualifizieren würden. Dies würde klare Anreize bei den Unternehmen setzen, Nachhaltigkeitsaspekte stärker als bisher in ihrer Strategie zu berücksichtigen. Letztlich kann eine Neugestaltung wichtiger Aktienindizes - auch über den DAX hinaus - einen Beitrag dazu leisten, dass mehr Kapital in nachhaltig wirtschaftende Unternehmen und Sektoren fließt.

\section{Literatur}

Deutsche Börse (2018), The DAX Index Universe, September.

Deutsche Börse (2019), Leitfaden zu den Aktienindizes der Deutsche Börse $A G$, Version 9.2.4, Juni.

Deutsche Börse (2020), Qualitätskriterien, internationale Standards, Transparenz: Marktkonsultation zur DAX-Reform gestartet, 5. Oktober.

FTSE Russel (2020), FTSE UK Index Series - Ground rules, September.

MSCl (2020), Introducing ESG Investing.

Qontigo, Deutsche Börse (2020a), STOXX calculation guide, Oktober.

Qontigo, Deutsche Börse (2020b), STOXX Index Methodology Guide (Portfolio-based Indices), Oktober.

Regierungskommission (2020), Deutscher Corporate Governance Kodex in der am 16. Dezember 2019 beschlossenen Fassung, März.

S\&P Dow Jones Indices (2019), Dow Jones Industrial Average Information, Januar.

Sustainalytics (2020), ESG Risk Ratings, August.

Title: More Sustainability in Germany's Leading Index DAX

Abstract: In the course of analysing the causes and consequences of the Wirecard scandal, a discussion about the potential reform of the German lead stock market index DAX is underway. The initial proposals presented by Deutsche Börse are moving in the right direction but are not farreaching enough. A clear signal is needed to indicate that future constituents of the DAX are obliged to meet at least a satisfactory level of sustainability, measured by an ESG (Environment, Social, Governance) Risk Score for the respective business activities. A simulation presented in this article demonstrates that some current members would no longer qualify for the DAX. Adding an ESG Risk Score to the current index selection criteria would set strong incentives for companies to gear their business strategies towards greater sustainability. Redesigning major stock indices to include sustainability aspects could ultimately play an important role in having more capital allocated to sustainably operating companies and sectors.

JEL Classification: G1, G2 